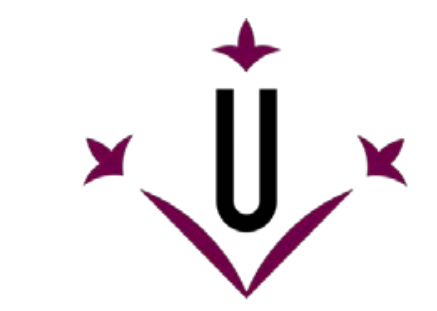

Universitat de Lleida

Document downloaded from:

http://hdl.handle.net/10459.1/65562

The final publication is available at:

https://doi.org/10.3920/WMJ2017.2282

Copyright

(c) Wageningen Academic Publishers, 2018 


\title{
Occurrence of Alternaria mycotoxins and quantification of viable Alternaria spp. during the food processing of tomato products in Spain
}

\author{
Estiarte, N. ${ }^{1}$, Crespo-Sempere, A. ${ }^{1,2}$, Marín, S. ${ }^{1}$, Sanchis, V. ${ }^{1}$ and Ramos, A.J. ${ }^{1 *}$
}

${ }^{1}$ Applied Mycology Unit, Food Technology Department. University of Lleida. UTPVXaRTA, Agrotecnio Center, Lleida, Spain.nestiarte@tecal.udl.cat; smarin@tecal.udl.cat; vsanchis@tecal.udl.cat; ajramos@tecal.udl.cat. ${ }^{2}$ Present address: Valgenetics S.L. University of Valencia Science Park, Valencia, Spain. acrespo@valgenetics.com.

*Corresponding author. Email: ajramos@tecal.udl.cat

\begin{abstract}
The occurrence of two Alternaria mycotoxins, alternariol (AOH) and alternariol monomethyl ether (AME) and the presence of conidia from Alternaria spp., were investigated throughout the food production chain of two businesses, one which uses organic fruit and the other nonorganic. For this purpose, a propidium monoazide (PMA) treatment followed by a quantitative Real Time PCR (qPCR) was used to detect and quantify viable conidia exclusively. Results demonstrated that $68.4 \%$ of the total raw fruit analysed was contaminated with viable Alternaria spp. Regarding the mycotoxin occurrence, only a few samples were contaminated with AME, while $35 \%$ of raw tomatoes tested positive for $\mathrm{AOH}$ in the organic producer and $21 \%$ in the non-organic producer. $\mathrm{AOH}$ was present in samples analysed before heat treatment, while almost no mycotoxins were found in the final products of the organic producer. However, in the non-organic producer, $47 \%$ of the tomato concentrates were contaminated.
\end{abstract}

Keywords: Alternaria spp., AOH, AME, Tomatoes

\section{Introduction}

The genus Alternaria includes several species that cause plant diseases in many crops and can spoil various fruits, grains, and vegetables during post-harvest storage and transport. Alternaria spp. are known to produce several secondary metabolites, some of which are mycotoxins. The most common mycotoxins associated with Alternaria in food commodities include alternariol $(\mathrm{AOH})$, alternariol monomethyl ether (AME), tentoxin (TEN), tenuazonic acid (TeA), altenuene (ALT) and altertoxins (Barkai-Golan, 2008; EFSA, 2011; Logrieco et al., 2009; Ostry, 2008).

The European Commission asked the European Food Safety Authority (EFSA) to assess the risk for animal and public health regarding Alternaria mycotoxin exposure. In this way, the European Commission and the competent authorities in the Member States could consider the need for a possible follow up, including filling knowledge gaps. In 2011, the EFSA emitted a scientific opinion in relation to the presence of Alternaria toxins in feed and food (EFSA, 2011). A total of 11730 analytical data were considered for this assessment, which included reports of the occurrence in food and feed and data published in scientific literature. Results were characterized by a high proportion of values below the limit of detection (LOD) and the limit of quantification (LOQ). The EFSA concluded there was a need for more representative occurrence data on Alternaria toxins in food and feed across the European countries in order to establish the real exposure by consumers. Additionally, the EFSA asked for more studies related to the influence of food and feed processing on Alternaria toxins. In 2016, the EFSA published a second scientific report analysing the dietary exposure to Alternaria toxins (AOH, AME, TeA and TEN) in the European population. Results demonstrated that TeA had the highest levels among the four Alternaria toxins assessed. The report also concluded that 'Infants' and 'Toddlers' were the age classes with the highest exposure and that vegetarians 
seem to have higher dietary exposure to Alternaria toxins than the general population (EFSA et al., 2016).

Tomatoes and their derived products are widely consumed all over the world but, unfortunately, due to their thin skin they are very susceptible to fungal decay. It has been reported that Alternaria spp. are the primary cause of black mould disease on raw tomatoes and are considered to be the major postharvest spoilers of fresh tomatoes (Andersen and Frisvad, 2004; Morris et al., 2000). While the direct consumption of a mouldy tomato by a consumer is unlikely, there is a possibility of mouldy tomatoes being used for the production of tomato products such as ketchups, purées, juices and sauces, among others. There are numerous published reports that describe the presence of Alternaria mycotoxins in different tomato commodities (Ackermann et al., 2011; da Motta and Valente Soares, 2001; Noser et al., 2011; Terminiello et al., 2006; Van de Perre et al., 2014; Visconti et al., 1987). Tomato fruit can be contaminated in crop fields and can enter the food industry with a high rate of contamination, which can become higher after a period of storage if conditions are favourable for fungal growth. Once inside the plant production, limited information is available regarding what happens to Alternaria toxins during storage and processing. There are some studies that indicate mycotoxins are highly stable and are not completely destroyed during food processing (Scott and Kanhere, 2001; Siegel et al., 2010). Another problem may be the manufacturing process itself. Commonly, in the food industry fruit is subject to two different immersions in water. The first occurs before entering the production plant and is used to wash fruit and transport it to manual sorting tables. The second immersion takes place after the manual selection, to allow the tomatoes to be transported more easily. The frequency of water replacement in these baths will depend on the business but it may be low. Hence, this water becomes highly contaminated and fruit may therefore be contaminated. Following the water bath, all tomatoes used for the production of purées or sauces go to a chopping tank where they are all chopped and mixed together. Hence, if food businesses use raw fruits contaminated with mycotoxin-producing Alternaria spp., these toxic compounds may be present in end products.

The Food and Agriculture Organization of the United Nations (FAO) specifies that all materials susceptible to contamination with natural toxins have to fulfil the levels indicated by national or international regulations (Dauthy, 1995). However, Alternaria mycotoxins are not regulated yet, and so no controls are required for these toxins. Only in some regions some regulations have been established to control Alternaria toxins (Rychlik et al., 2016).

The aim of this study was to investigate what happens to conidia from Alternaria spp. throughout the industrial food chain. For this purpose, propidium monoazide (PMA) treatment, a DNA binding dye that allows the detection of viable conidia exclusively, was used in a quantitative Real Time PCR (qPCR) to quantify the viable conidia present in each of the samples analysed. Additionally, the occurrence of AOH and AME was assessed in several production stages of both organic and non-organic tomato processing plants. Results may indicate that regulations are needed for these toxic contaminants.

\section{Material and methods}

\subsection{Analytical standards and chemicals}

Standards of AOH ( $\sim 96 \%)$ and AME ( 96\%) were supplied by Sigma-Aldrich (Alcobendas, Spain). A stock solution was prepared for each standard by dissolving $5 \mathrm{mg}$ of the purified mycotoxins in ethanol, reaching a final concentration of $1000 \mu \mathrm{g} / \mathrm{mL}$. From the stock standard solutions, working standard solutions at a concentration of $15 \mu \mathrm{g} / \mathrm{mL}$ were prepared. AOH and AME concentration was checked by UV spectroscopy. All standards were stored at $-20^{\circ} \mathrm{C}$ in sealed vials until use.

Acetonitrile (99.9\%) and methanol (99.9\%) were both HPLC (high-performance liquid chromatography) grade and were supplied by J.T. Baker (Deventer, The Netherlands). Pure water was obtained from a milli-Q apparatus (Millipore, Billerica, MA, USA). 
Tomato samples were collected from two different food producers, during two harvest seasons, 2012/2013 and 2013/2014. Both producers were located in the province of Lleida (NE Spain). One of the producers worked with fresh organic tomatoes while the other used non-organic tomatoes. Samples were collected randomly at all production stages where it was physically possible, since in some stages the process was completely automated and samples could not be collected (Fig. 1). In the organic plant, raw tomatoes were collected randomly from wooden pallets before they entered the production plant. Once inside the plant, samples were taken before heat treatment (peeled tomatoes and mashed tomatoes). Waste by-products, such as rotten tomatoes that had been rejected during the manual selection were also collected. Tomato skin peels, obtained from the production of scalded peeled tomatoes, were sampled as well. The final products analysed from the organic plant were tomato purée and peeled tomatoes. From the non-organic plant, raw tomatoes were collected randomly before entering the production plant and before heat treatment (mashed tomatoes). In this case, the final product analysed was tomato concentrate. In both cases, samples were collected randomly on different days during the season. To collect each sample, $\sim 1 \mathrm{~kg}$ of tomato source was taken at different stages and collected in plastic bags. Once in the laboratory, samples were properly weighted and sampled as explained in section 2.3.1.

\subsection{Quantification of viable Alternaria conidia}

\subsubsection{Sample preparation}

Once in the laboratory, samples were weighted $(1 \mathrm{~kg})$. Half of the total sample was mixed with one volume of phosphate buffer saline (PBS, $138 \mathrm{mM} \mathrm{NaCl}, 2.7 \mathrm{mM} \mathrm{KCl}, 10 \mathrm{mM}$ $\mathrm{NaH}_{2} \mathrm{PO}_{4} \cdot 2 \mathrm{H}_{2} \mathrm{O}$, and $2 \mathrm{mM} \mathrm{KH} \mathrm{PO}_{4}$ ) and liquidised in a blender (Turbo Habana, Palson, Spain). This step was repeated twice with the other half of the sample and, at the end, the whole sample was homogenised in a bigger beaker. From this mixture, two aliquots of $50 \mathrm{~mL}$ were collected for the analysis of $\mathrm{AOH}$ and $\mathrm{AME}$ (see section 2.4). These samples were frozen at $-20{ }^{\circ} \mathrm{C}$ until performing the mycotoxin extraction and the HPLC analysis. Similarly, for assessing the presence of viable conidia of Alternaria spp., two aliquots of $50 \mathrm{~mL}$ were taken from the sample and DNA extraction was carried out immediately.

\subsubsection{DNA quantification from viable conidia using the PMA-qPCR technique}

For this assay, DNA extraction and quantification was performed from each $50-\mathrm{mL}$ aliquot collected during sample preparation (see section 2.3.1). Hence, each tomato sample collected from the plant was analysed in duplicate. Tomato samples were filtered through a Miracloth paper (Calbiochem, USA) and centrifuged at $15000 \times \mathrm{g}$ for $10 \mathrm{~min}$. Pellets were resuspended in $2 \mathrm{~mL}$ of PBS. Next, the PMA treatment was carried out combined with the qPCR (PMAqPCR) to quantify viable conidia from Alternaria spp. The PMA treatment, DNA extraction and $\mathrm{qPCR}$ detection and quantification were performed as detailed in Crespo-Sempere et al. (2013). In the current study, the LOD was deemed to be the lowest DNA concentration that can be detected with 95 per cent confidence that it is a true detection. For its calculation, a six-point calibration curve was developed using five different conidia concentrations of $A$. alternata $\left(10,10^{2}, 10^{3}, 10^{4}\right.$ and $10^{5}$ conidia/g of tomato), which were used to artificially contaminate different samples of tomato purée. Non-contaminated tomato purée was considered a negative control. Next, the PMA-qPCR method was applied to these contaminated tomato purée samples. A five-point calibration curve was developed using the A. alternata DNA extracted from each one of the tomato samples (Fig. 2). The mean of the quantification cycles $(\mathrm{Cq})$ of all the replicates with the lowest DNA concentration (10 conidia/g of tomato) was used to calculate the LOD by using the following equation:

$$
\log _{10} \text { Concentration }=\frac{C q-b}{m}
$$


Where $m$ is the slope of the regression line and $b$ is the interception point with the y-axis (Fig. 2). According to Eq. 1, the LOD was deemed to be 11 conidia/g of tomato. The LOQ was deemed to be the lowest concentration that could be quantified reliably. The Cq of the LOQ was determined using the following equation:

$$
C q_{L O Q}=C q_{L O D}-2\left(\sigma_{L O D}\right)
$$

Equation 2 Where $\mathrm{Cq}_{\mathrm{LOD}}$ was the average of all the replicates with the lowest DNA concentration detectable and $\sigma$ their standard deviation. According to Eq. 1 and Eq. 2, the LOQ established for this work was 28 conidia/g of tomato. Once the LOD and the LOQ were determined, all values below the LOD were considered 0 , while values between the LOD and the LOQ were substituted by 28 conidia/g of tomato.

\subsection{Assessment of natural occurrence of $A O H$ and $A M E$ \\ 2.4.1 AOH and AME extraction}

$\mathrm{AOH}$ and $\mathrm{AME}$ extraction was done from the two different $50-\mathrm{mL}$ aliquots collected during sample preparation once in the lab (section 2.3.1). Hence, each tomato sample collected from the plant was analysed in duplicate. For the AOH and AME extraction procedure, $20 \mathrm{~g}$ of each tomato sample was mixed with $60 \mathrm{~mL}$ of acetonitrile-methanol-water $(45: 10: 45 \mathrm{v} / \mathrm{v} / \mathrm{v}$; $\mathrm{pH} 3$ adjusted with $o$-phosphoric acid) in a small glass beaker and homogenized for 15 minutes using a uniform magnetic mixer. The solution was left for approximately 10 minutes, to favour precipitation by gravity. Next, $6 \mathrm{~mL}$ of the supernatant was transferred to a centrifuge tube and diluted with $15 \mathrm{~mL}$ of a $0.05 \mathrm{M}$ sodium dihydrogen phosphate solution (pH 3 adjusted with $o$-phosphoric acid) and centrifuged at $15250 \times \mathrm{g}$ for 10 minutes. After that, $2 \mathrm{~mL}$ of the diluted sample extract was passed by gravity filtration through a previously conditioned Bond Elut Plexa SPE cartridge ( $200 \mathrm{mg}$ and $6 \mathrm{~mL}$, Agilent Technologies, Santa Clara, CA, USA). Conditioning of the cartridge was done with $5 \mathrm{~mL}$ of methanol followed by $5 \mathrm{~mL}$ of miliQ water. The SPE column was washed with $5 \mathrm{~mL}$ of water followed by air drying on the manifold. Finally, elution was carried out with $5 \mathrm{~mL}$ of methanol and $5 \mathrm{~mL}$ of acetonitrile. Sample extracts were dried in a speed vacuum concentrator at room temperature and stored at $-20{ }^{\circ} \mathrm{C}$ until the HPLC analysis. Prior to HPLC injection, samples were resuspended in $500 \mu \mathrm{L}$ of a water-methanol solution $(50: 50 \mathrm{v} / \mathrm{v})$ and sonicated for 1 minute.

\subsubsection{HPLC analysis}

Separation, detection and quantification of $\mathrm{AOH}$ and AME were performed on a HPLC system consisting of a Waters 2695 Alliance Separations Module connected to a UV/Visible dual $\lambda$ Waters Absorbance Detector 2487, using a reversed phase Kinetex PFP column ( $5 \mu \mathrm{m}$, $4.6 \times 150 \mathrm{~mm}$, Phenomenex, Torrance, CA, USA) preceded by a Spherisorb guard column $(5 \mu \mathrm{m}$ ODS2, $4.6 \times 10 \mathrm{~mm}$, Waters, Millford, MA, USA). Chromatographic conditions are deseribed in Estiarte et al. (2016). For mycotoxin quantification, working standard solutions were used to perform a ten-point calibration curve $(1500,1250,1000,750,500,250,100,50$, 25 and $10 \mathrm{ng} / \mathrm{mL}$ ). The LOD of the analysis was $10 \mu \mathrm{g} / \mathrm{kg}$ of tomato for AOH and $12 \mu \mathrm{g} / \mathrm{kg}$ of tomato for AME, based on a signal-to-noise ratio of 3:1. The LOQ was calculated as $3 \times$ LOD. All solvents were HPLC grade and all chemicals were analytical grade. Method performance characteristics for AOH and AME are detailed in Estiarte et al. (2016).

2.5 Statistical analysis

All statistical data were analysed assuming a non-parametric distribution. Multiple comparisons were made with the Wilcoxon test, which compares the medians between pairs. The p-value was established as 0.05. All statistical analyses were performed with the JMP program.

\section{Results and discussion}

\subsection{Presence of viable Alternaria spp.}


The primer set Alt4-Alt5 used in this study was designed previously to detect and quantify, with the qPCR, several Alternaria spp. such as A. alternata, A. arborescens, A. tenuissima, A. tomato, A. tomatophila, A. tomaticola and A. solani (Crespo-Sempere et al., 2013). Primer set Alt4-Alt5 was not able to distinguish between Alternaria spp. and Ulocladium botrytis, but the joint detection of both genera could be an advantage for the food industry since $U$. botrytis is also considered a plant pathogen and a mycotoxin producer (Andersen and Hollensted, 2008). All these species are commonly associated with the decay of fruits and vegetables, especially in tomatoes. For this study, a total of 175 samples were analysed between 2012 and 2014. 115 of these samples were collected from a business using organic tomatoes as raw fruit (Fig. 3). The other 60 tomato samples were taken from a food business that used non-organic raw tomatoes (Fig. 4). The PMA-qPCR technique allows detecting exclusively the viable conidia. Results showed that $72.6 \%$ of samples were contaminated with viable Alternaria. Among these samples, $24.4 \%$ had an amount of contamination below the LOQ (28 conidia/g of tomato). Within the positive samples, 73 samples $(57.5 \%)$ corresponded to the organic plant, while $54(42.5 \%)$ had been collected from the non-organic company. The box plots illustrated in Figs. 3 and 4 show the distribution of fungal concentration for all the production stages analysed.

Several production stages were analysed for the presence of viable Alternaria spp. in the organic plant. From the whole raw tomato fruit collected, $60.4 \%$ was positive for viable conidia. Regarding the non-organic company, $80.6 \%$ of the raw tomatoes were contaminated with viable conidia. While the non-organic company had the highest percentage value of raw sampled contaminated with viable conidia, the median for both was very similar and the statistical analysis did not show significant differences ( $p$-value $>0.05$ ). Importantly, in both businesses it was observed that the contamination with Alternaria was very heterogeneous. Samples were collected in different days during two seasons and, depending on the day, the ripeness of fruit and the percentage of rotten tomatoes was different, which may affect the total contamination of Alternaria spp. In fact, warm rainy weather or dew formation on the fruit surface favours Alternaria disease. Additionally, depending on the ripeness of the fruit, infection may be more severe. The riper the fruit is, the more susceptible it is to fruit decay (Logrieco et al., 2003). All these variables may influence fungal concentration levels. Despite the variability of Alternaria concentration, black mould disease was observed in many tomatoes when collecting samples randomly. In fact, it has been reported that Alternaria spp. are the primary cause of black mould disease in raw tomatoes (Morris et al., 2000). Reports on the occurrence of Alternaria spp. in fruits are numerous, especially in tomatoes. Harwig et al. (1979) studied which moulds were present in rotten tomatoes and observed that 15 out of 41 were Alternaria spp., which represents $37 \%$. These results were supported some years later by Andersen and Frisvad (2004), who isolated fungal colonies from several mouldy fresh tomatoes, home-grown and tomatoes from supermarkets. They found that the most predominant genus was Alternaria (40\%), followed by Penicillium (25\%), Stemphylium (15\%), and Cladosporium (10\%). Among Alternaria spp., A. tenuissima was the most frequent species. More recently, Pavón et al. (2012a) detected by PCR the presence of Alternaria spp. in raw and processed commercial tomato samples. Results stated that raw samples $(57.5 \%)$ and processed products $(60 \%)$ were contaminated with Alternaria. These percentages are very similar to results obtained in this work. Regarding tomato products they found that 41 out of 90 commercial samples $(45.6 \%)$ were contaminated with Alternaria spp. (Pavón et al., 2012b).

Before entering in the production plant, tomatoes are washed in a water bath. Afterwards, rotten tomatoes are manually sorted in sorting tables and rotten or mouldy tomatoes are rejected. In the organic business, these rejected tomatoes were assessed for the presence of Alternaria and it was observed that $81.8 \%$ of the samples were contaminated with Alternaria conidia. However, even though tomatoes had been washed in a water bath and selected manually, the concentration of viable Alternaria did not decrease at all on the following 
production stages. In the non-organic business, the same tendency was observed. The Wilcoxon test showed that mashed and rejected tomatoes were significantly different compared to the rest ( $\mathrm{p}$-value $<0.05$ ). Mashed tomatoes had higher concentrations of viable Alternaria compared to raw tomatoes. A possible explanation could be the immersion of raw fruit in water baths. Commonly, in the food industry, there are two different immersions in water. The frequency of water replacement in these baths will depend on the food industry but it may be low. Hence, this water becomes highly contaminated and fruit may therefore become contaminated. Following the water bath, all tomatoes used for the production of purées or sauces go to a chopping tank where they are chopped and mixed together. The mashed tomatoes analysed in this study were collected from this tank. It may be important to note that, when producing scalded peeled tomatoes the contamination was lower. In this case, tomatoes are not all blended together and the skin is removed. Additionally, for the production of scalded peeled tomatoes the quality of raw fruit is usually better and, probably, less contaminated with fungi. Indeed, in assessing fungal contamination of tomato skin samples, results showed that these samples were not contaminated at all as the median was below our LOD. All these factors together may help to decrease the total fungal contamination when producing scalded peeled tomatoes.

The presence of Alternaria conidia was not analysed in the final products as it was assumed that fungi would be killed during the heat treatment and so, no viable conidia would be found.

\subsection{Occurrence of $A O H$ and $A M E$ along the food production chain}

A total of 277 tomato samples were analysed for the presence of both AOH and AME during two different harvest seasons. Of these samples, 184 were collected from the organic plant, and 93 from the non-organic plant. As previously done for the assessment of Alternaria, samples were collected from different stages of the industrial production chain. In general, the occurrence of $\mathrm{AOH}$ was far higher than AME, as just 5 out of 277 tomatoes were contaminated with AME. All samples contaminated with AME were collected from the organic plant (Table 1). As described in Table 1, the group of samples that reached the highest percentage of contamination with $\mathrm{AOH}$ in the organic industry were the set of samples of rejected tomatoes $(40 \%)$, followed by tomato skin (36\%) and raw fruits (35\%). The two final products analysed, scalded peeled tomatoes and tomato purée, were not contaminated at all. Just one sample of purée was found to be positive. In fact, it is reasonable that the highest percentage of contamination for $\mathrm{AOH}$ is found among rejected samples because these fruits were rotten and they were discarded from the production flow due to their high fungal contamination (see section 3.1). In contrast, the contamination of AOH (median, $96.5 \mu \mathrm{g} / \mathrm{kg}$ ) or AME (median, $103.3 \mu \mathrm{g} / \mathrm{kg}$ ) in mashed tomatoes, which were highly contaminated with Alternaria conidia, was not high at all. This could be explained by the fact that the contamination of mashed and peeled tomatoes possibly takes place when tomatoes are dipped into water during the washing step. Hence, these Alternaria spp. did not have time to biosynthesize mycotoxins since samples were collected immediately after the washing step. In the non-organic plant (Table 2), results showed that considering only positive samples, the median of contamination for AOH in raw fruit $(1165.2 \mu \mathrm{g} / \mathrm{kg}$ of tomato) was higher than the one in the organic industry $(261.4 \mu \mathrm{g} / \mathrm{kg}$ of tomato). Comparing mashed tomatoes from the organic and non-organic plants, no significant differences were found (p-value $>0.05$ ). In both plants, the assessment of the presence of $\mathrm{AOH}$ and AME in different production stages showed that toxin levels decreased throughout the production chain. However, in the nonorganic plant, $47 \%$ of the final products assessed were significantly more contaminated with $\mathrm{AOH}$ with a median of $39 \mu \mathrm{g} / \mathrm{kg}$. By contrast, the final products from the organic industry almost no contaminated samples were found (just one positive sample, $26 \mu \mathrm{g} / \mathrm{kg}$ ). It is important to mention that the final product analysed was not the same (scalded peeled tomatoes, tomato purée and tomato concentrate) and the food process may influence the final concentration in some way. 
Terminiello et al. (2006) investigated the presence of AOH, AME and TeA in 80 samples of tomato purée. Thirty-nine out of 80 samples were contaminated with Alternaria mycotoxins. Levels of $\mathrm{AOH}$ ranged from 187 to $8756 \mu \mathrm{g} / \mathrm{kg}$, for AME it was 84 to $1734 \mu \mathrm{g} / \mathrm{kg}$ and for TeA, 39 to $4021 \mu \mathrm{g} / \mathrm{kg}$. Cereals, fruit and vegetable products have also been analysed for $\mathrm{AOH}$ and AME contamination by Asam et al. (2010). Both toxins were frequently detected in vegetable products. The authors found that $\mathrm{AOH}$ levels ranged from 2.6 to $25 \mu \mathrm{g} / \mathrm{kg}$, while for AME it was 0.1 to $5 \mu \mathrm{g} / \mathrm{kg}$. Indeed, they reported that tomato products were especially affected. Ackermann et al. (2011) also found the presence of AOH in 93\% of samples of tomato products. From this work and the existent literature, it can be said that Alternaria mycotoxins are present in food products. However, their concentration is fairly variable and it may depend on weather conditions and food processing. It is important to mention that in Europe, there is some official regulation for Alternaria mycotoxins (Rychlik et al., 2016), but these toxic products could be better regulated and dietary exposure could at least be controlled.

\section{Conclusions}

In conclusion, there is evidence of the presence of Alternaria spp. in tomatoes used for the production of tomato derived products, since $68.4 \%$ of raw samples were contaminated with viable Alternaria spp. Of all these contaminated samples, $75.6 \%$ had levels of contamination above 28 conidia/g of tomato. Maximum levels reached almost 2000 conidia/g of tomato in both organic and non-organic industries. Therefore, controlling the water used for washing and a better selection of the raw fruits may be good preventive measures to reduce the contamination of tomatoes. Additionally, the presence AOH and AME has been demonstrated. The levels of both toxins decreased throughout the production process of tomato products. Nevertheless, $47 \%$ of the final product of one of the companies was contaminated with $\mathrm{AOH}$, which may represent a significant risk to human health. Considering that Alternaria is the fungus most frequently responsible for tomato fruit decay, the assessment of the presence of Alternaria DNA or their mycotoxins may be considered a good parameter to determine the quality of the raw material that enters food production. However, the lack of standardized protocols for this kind of analysis may be an important limiting factor.

\section{Acknowledgements}

We thank both companies for their collaboration in this study and for opening their doors to us. We also thank Montse Prim for kindly helping us with the HPLC analysis of samples and Arthur Kendall for correction of English usage and style. We are grateful to the Catalonian Government (XaRTA-Reference Network on Food Technology) and the University of Lleida for their financial support. N. Estiarte thanks the Secretaria de Universitats i Recerca del Departament de Economia i Coneixement of the Generalitat de Catalunya for the pre-doctoral grant (FI-DGR 2013).

\section{References}

Ackermann, Y., Curtui, V., Dietrich, R., Gross, M., Latif, H., Märtlbauer, E. and Usleber, E., 2011. Widespread occurrence of low levels of alternariol in apple and tomato products, as determined by comparative immunochemical assessment using monoclonal and polyclonal antibodies. Journal of Agricultural and Food Chemistry 59: 6360-6368. 
Andersen, B. and Frisvad, J.C., 2004. Natural occurrence of fungi and fungal metabolites in moldy tomatoes. Journal of Agricultural and Food Chemistry 52: 7507-7513.

Andersen, B. and Hollensted, M., 2008. Metabolite production by different Ulocladium species. International Journal of Food Microbiology 126: 172-179.

Asam, S., Konitzer, K. and Rychlik, M., 2010. Precise determination of the Alternaria mycotoxins alternariol and alternariol monomethyl ether in cereal, fruit and vegetable products using stable isotope dilution assays. Mycotoxin Research 27: 23-28.

Barkai-Golan, R., 2008. Chapter 8 - Alternaria mycotoxins. In: R. Barkai-Golan and N. Paster (Eds.), Mycotoxins in Fruits and Vegetables. Academic Press, San Diego, pp. 185-203.

Crespo-Sempere, A., Estiarte, N., Marín, S., Sanchis, V. and Ramos, A.J., 2013. Propidium monoazide combined with real-time quantitative PCR to quantify viable Alternaria spp. contamination in tomato products. International Journal of Food Microbiology 165: 214-220.

da Motta, S. and Valente Soares, L.M., 2001. Survey of Brazilian tomato products for alternariol, alternariol monomethyl ether, tenuazonic acid and cyclopiazonic acid. Food Additives \& Contaminants 18: 630-634.

Dauthy, M.E., 1995. Fruit and vegetable processing, FAO Agricultural Services Bulletin No.119. Food and Agriculture Organization of the United Nations, Rome.

EFSA, 2011. Scientific opinion on the risks for animal and public health related to the presence of Alternaria toxins in feed and food. EFSA Journal 9: 2407-2504.

EFSA, Arcella, D., Eskola, M. and Gómez Ruiz, J., 2016. Scientific report on the dietary exposure assessment to Alternaria toxins in the European population. EFSA Journal 2016 14: 1831-4732.

Estiarte, N., Crespo-Sempere, A., Marín, S., Sanchis, V. and Ramos, A.J., 2016. Effect of 1methylcyclopropene on the development of black mold disease and its potential effect on alternariol and alternariol monomethyl ether biosynthesis on tomatoes infected with Alternaria alternata. International Journal of Food Microbiology 236: 74-82.

Harwig, J., Scott, P.M., Stoltz, D.R. and Blanchfield, B.J., 1979. Toxins of molds from decaying tomato fruit. Applied and Environmental Microbiology 38: 267-274. 
Logrieco, A., Bottalico, A., Mulé, G., Moretti, A. and Perrone, G., 2003. Epidemiology of toxigenic fungi and their associated mycotoxins for some Mediterranean crops European Journal of Plant Pathology 109: 645-667.

Logrieco, A., Moretti, A. and Solfrizzo, M., 2009. Alternaria toxins and plant diseases: an overview of origin, occurrence and risks. World Mycotoxin Journal 2: 129-140.

Morris, P.F., Connolly, M.S. and St Clair, D.A., 2000. Genetic diversity of Alternaria alternata isolated from tomato in California assessed using RAPDs. Mycological Research 104: 286-292.

Noser, J., Schneider, P., Rother, M. and Schmutz, H., 2011. Determination of six Alternaria toxins with UPLC-MS/MS and their occurrence in tomatoes and tomato products from the Swiss market. Mycotoxin Research 27: 265-271.

Ostry, V., 2008. Alternaria mycotoxins: an overview of chemical characterization, producers, toxicity, analysis and occurrence in foodstuffs. World Mycotoxin Journal 1: 175-188.

Pavón, M.Á., González, I., Martín, R. and García Lacarra, T., 2012a. ITS-based detection and quantification of Alternaria spp. in raw and processed vegetables by real-time quantitative PCR. Food Microbiology 32: 165-171.

Pavón, M.Á., Luna, A., de la Cruz, S., González, I., Martín, R. and García, T., 2012b. PCRbased assay for the detection of Alternaria species and correlation with HPLC determination of altenuene, alternariol and alternariol monomethyl ether production in tomato products. Food Control 25: 45-52.

Rychlik, M., Lepper, H., Weidner, C. and Asam, S., 2016. Risk evaluation of the Alternaria mycotoxin tenuazonic acid in foods for adults and infants and subsequent risk management. Food Control 68: 181-185.

Scott, P.M. and Kanhere, S.R., 2001. Stability of Alternaria toxins in fruit juices and wine. Mycotoxin Research 17: 9-14.

Siegel, D., Feist, M., Proske, M., Koch, M. and Nehls, I., 2010. Degradation of the Alternaria mycotoxins alternariol, alternariol monomethyl ether, and altenuene upon bread baking. Journal of Agricultural and Food Chemistry 58: 9622-9630. 
412 Terminiello, L., Patriarca, A., Pose, G. and Fernandez Pinto, V., 2006. Occurrence of 413 alternariol, alternariol monomethyl ether and tenuazonic acid in Argentinean tomato puree. 414 Mycotoxin Research 22: 236-240.

415 Van de Perre, E., Deschuyffeleer, N., Jacxsens, L., Vekeman, F., Van Der Hauwaert, W., 416 Asam, S., Rychlik, M., Devlieghere, F. and De Meulenaer, B., 2014. Screening of moulds and 417 mycotoxins in tomatoes, bell peppers, onions, soft red fruits and derived tomato products. 418 Food Control 37: 165-170.

419 Visconti, A., Logrieco, A., Vurro, M. and Bottalico, A., 1987. Tenuazonic acid in blackmold 420 tomatoes: occurrence, production by associated Alternaria species, and phytotoxic properties. 421 Phytopathologia Mediterranea 26: 125-128. 


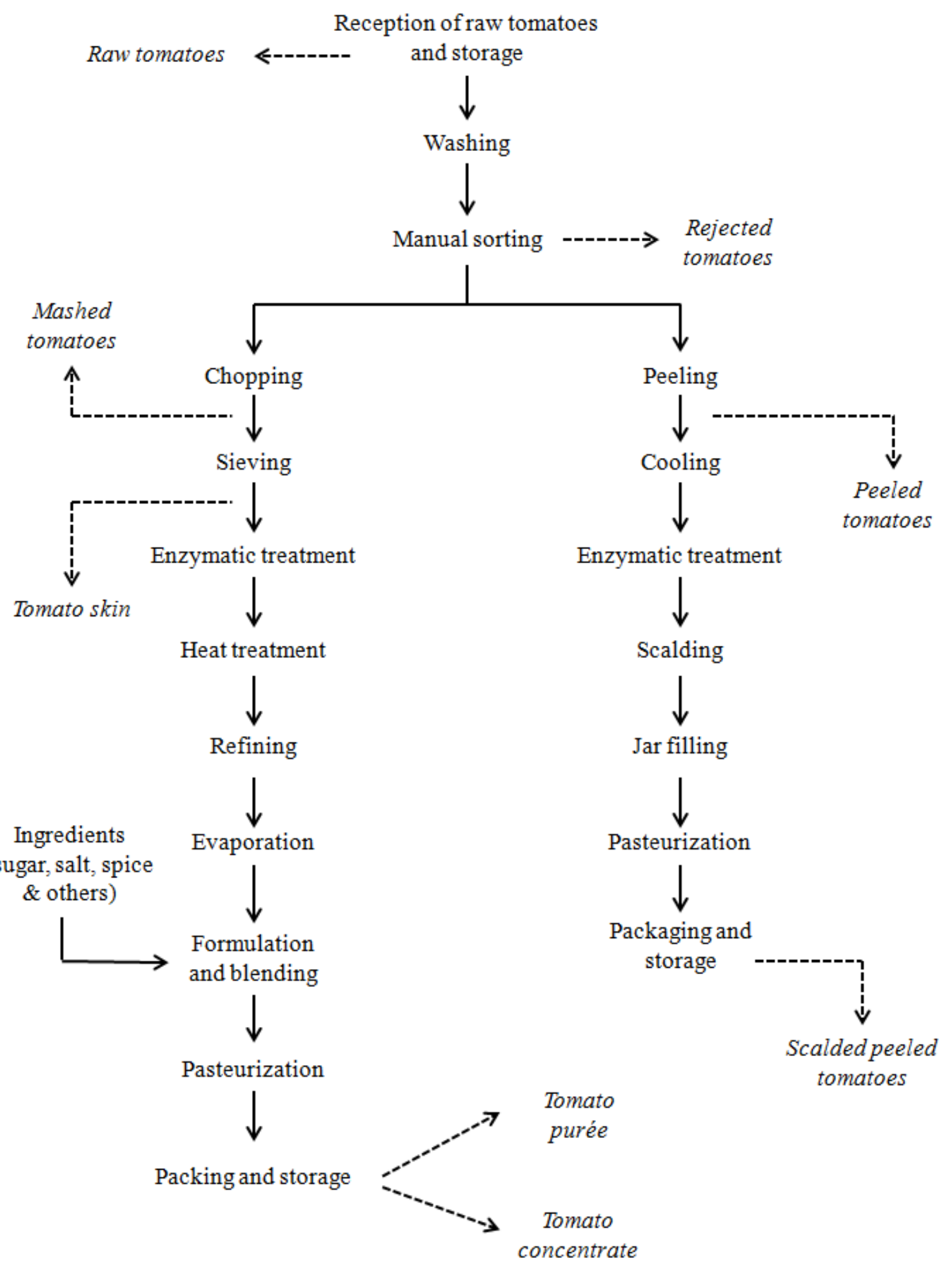

Figure 1. Production flow chart of derived tomato products. Italic letters specify the type of tomato samples that were collected in the organic and conventional industry for the assessment of Alternaria spp. presence and occurrence of AOH and AME. 


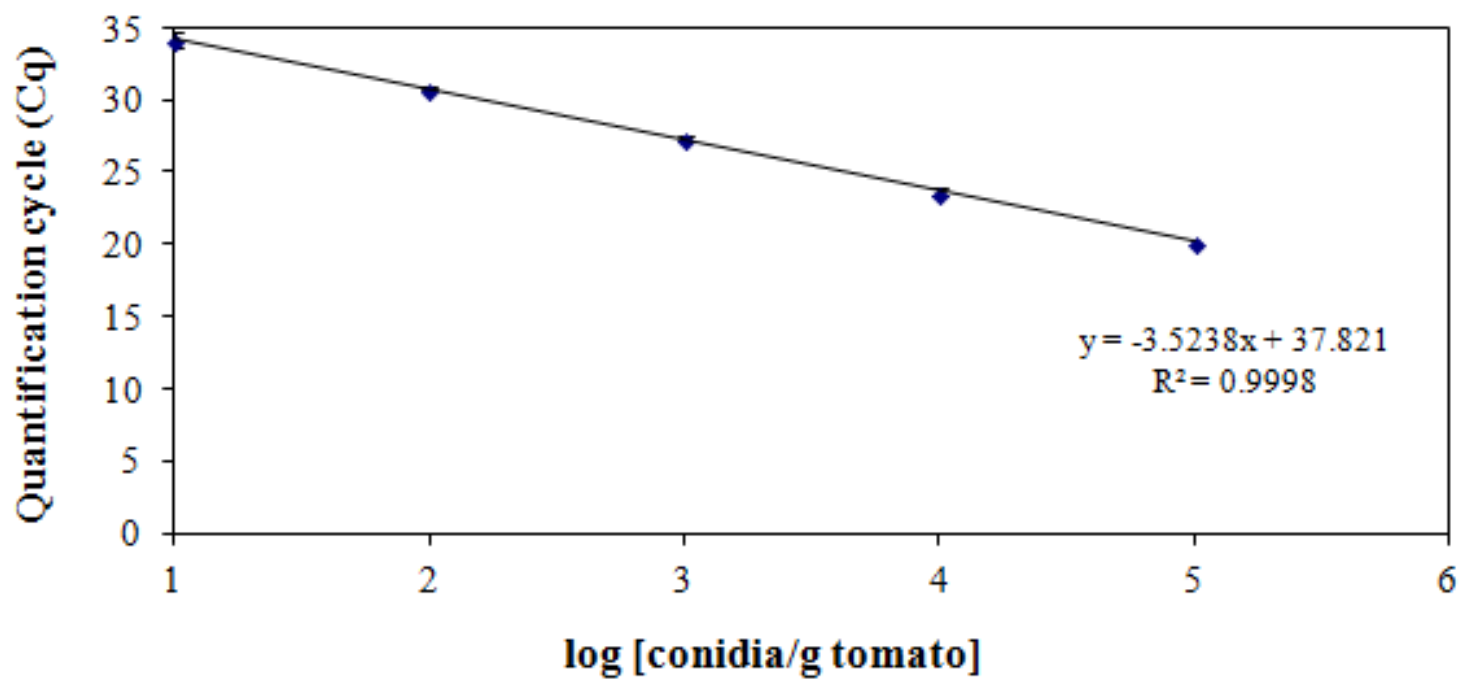

430

431

432

433

434

435

Figure 2. Standard curve obtained with SYBR Green I using five tomato food matrix samples artificially inoculated with $A$. alternata conidia with different concentration each sample $\left(10^{5}\right.$, $10^{4}, 10^{3}, 10^{2}$ and 10 conidia/g of tomato). 


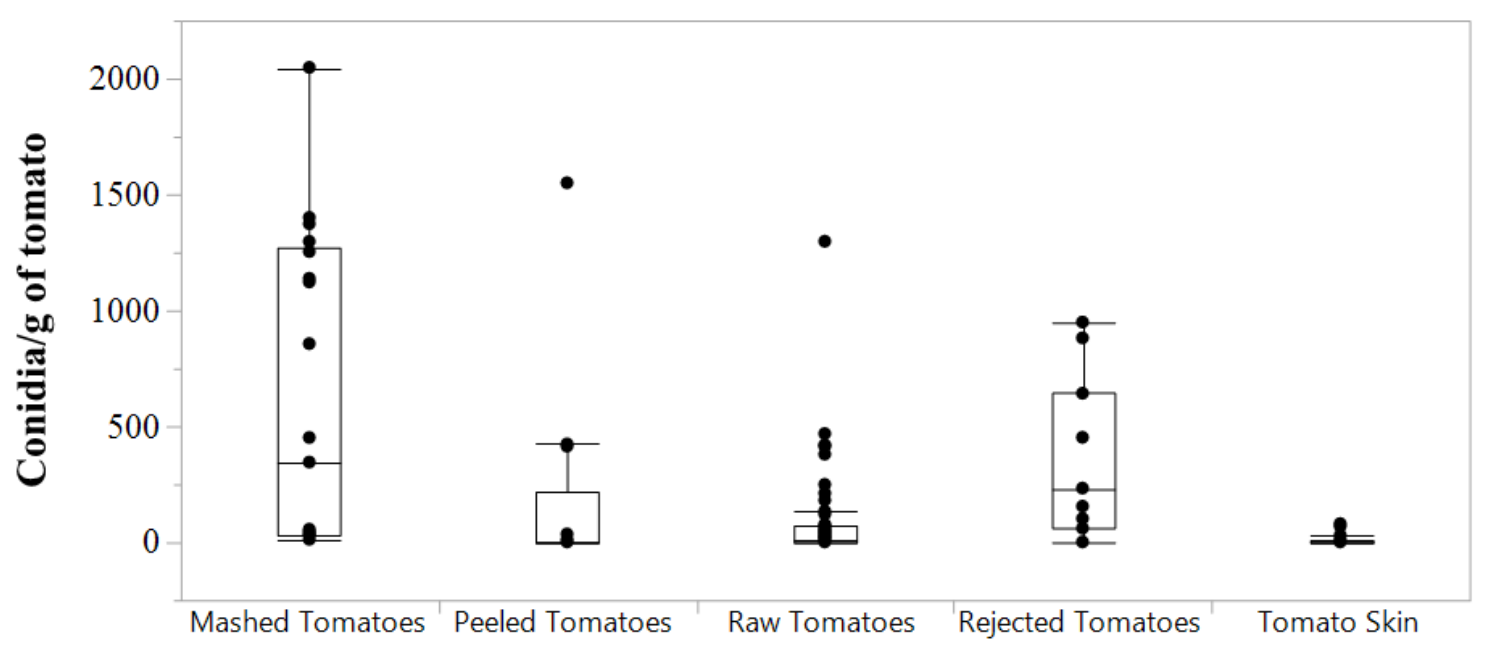

\section{Type of Samples}

436

Figure 3. Distribution of the concentration of viable Alternaria conidia during the tomato processing in an industry with an organic production. The horizontal line within the box represents the median sample value. The ends of the box represent the 3rd and 1st quartile. The whiskers represent the lowest datum still within 1.5xIQR of the lower quartile, and the highest datum still within 1.5xIQR of the upper quartile.

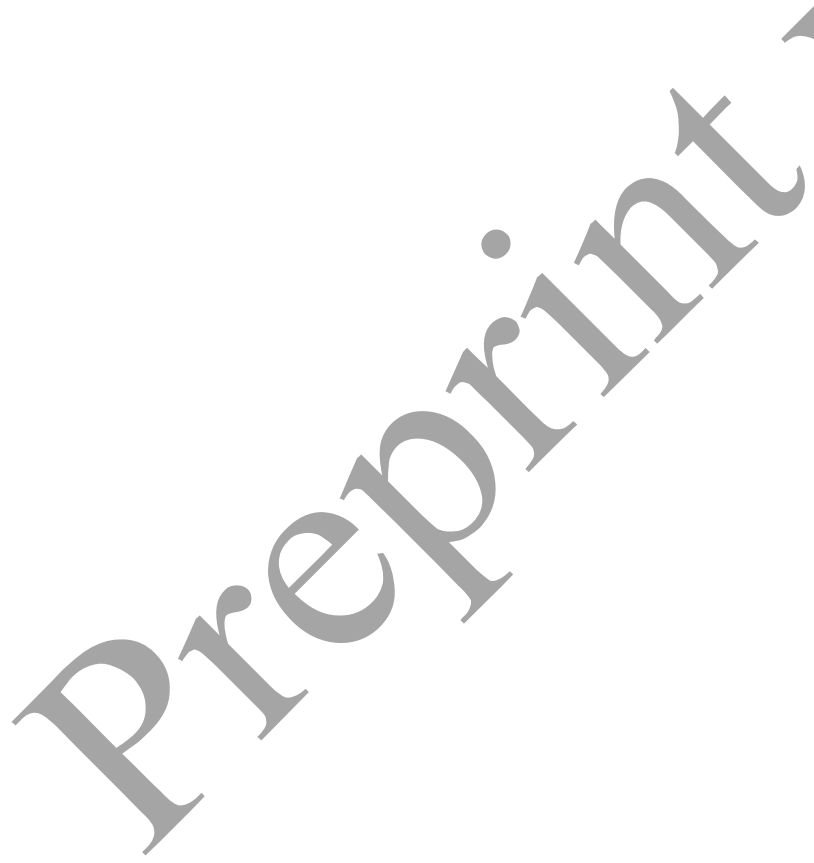




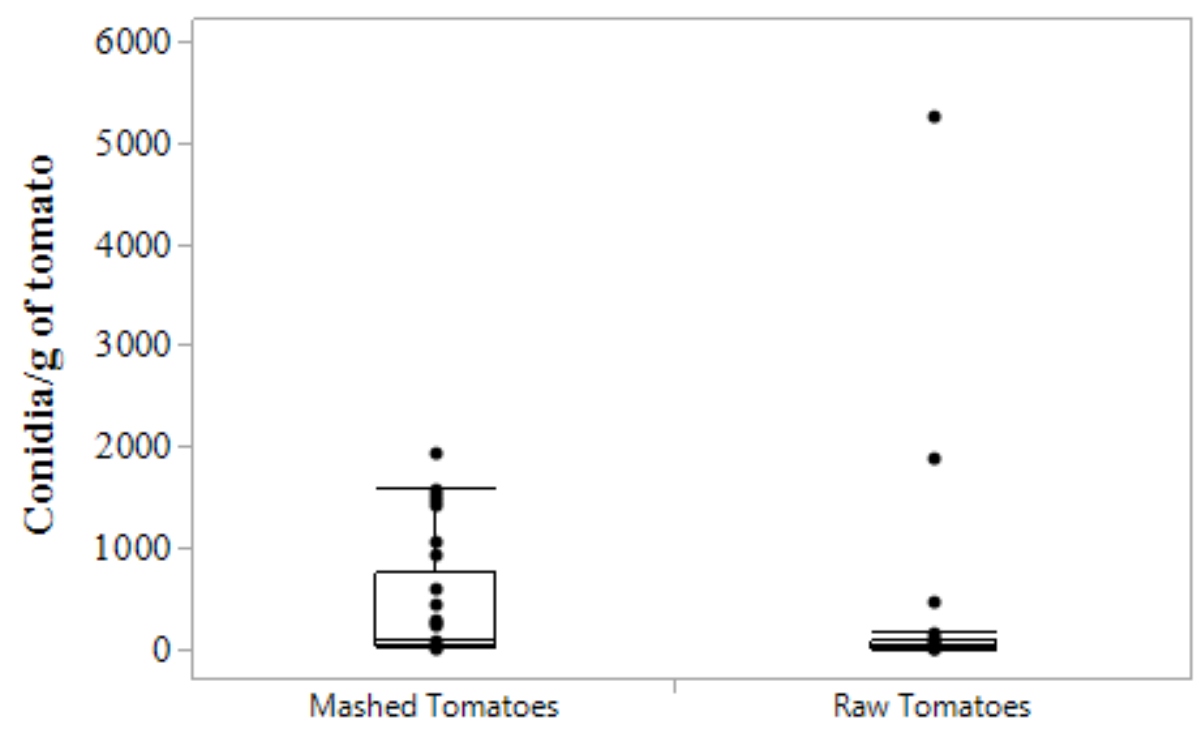

Type of Samples

Figure 4. Distribution of the concentration of viable Alternaria conidia during the tomato processing in an industry with a conventional production. The horizontal line within the box represents the median sample value. The ends of the box represent the 3 rd and 1st quartile. The whiskers represent the lowest datum still within 1.5xIQR of the lower quartile, and the highest datum still within $1.5 \times \mathrm{IQR}$ of the upper quartile. 


\begin{tabular}{ccccccccc} 
& \multicolumn{3}{c}{ AOH } & & & \multicolumn{3}{c}{ AME } \\
\cline { 2 - 9 } n & $\begin{array}{c}\text { Positive } \\
\text { Samples (\%) }\end{array}$ & Range* & Mean* & Median* & $\begin{array}{c}\text { Positive } \\
\text { Samples (\%) }\end{array}$ & Range* & Mean* & Median* \\
\hline 48 & $17(35 \%)$ & $95.4-1318.6$ & 386.8 & 261.4 & $1(2 \%, 136.8)$ & - & - & - \\
18 & $4(22 \%)$ & $117.4-445.5$ & 269.4 & 257.4 & 0 & ND & ND & ND \\
20 & $3(15 \%)$ & $95.4-100.3$ & 97.4 & 96.5 & $2(10 \%)$ & $100.9-105.6$ & 103.2 & 103.2 \\
14 & $5(36 \%)$ & $153.1-209.8$ & 169.5 & 162.6 & $1(7 \%, 502.2)$ & - & - & - \\
10 & $4(40 \%)$ & $111.2-265.3$ & 165.2 & 142.1 & $1(2 \%, 197.8)$ & - & - & - \\
51 & 0 & ND & ND & ND & - & ND & ND & ND \\
23 & $1(4 \%, 26.4)$ & - & - & - & 0 & ND & ND & ND
\end{tabular}

Raw tomatoes

Peeled tomatoes

Mashed tomatoes

Tomato skin

Rejected tomatoes

Scalded peeled tomatoes

Tomato purée

Table 1.

* The range, the mean and the median were calculated just for positive samples. Units for range, mean and median were $\mu \mathrm{g} / \mathrm{kg}$ of tomato. When only one sample was positive, the concentration value is specified next to the percentage value.

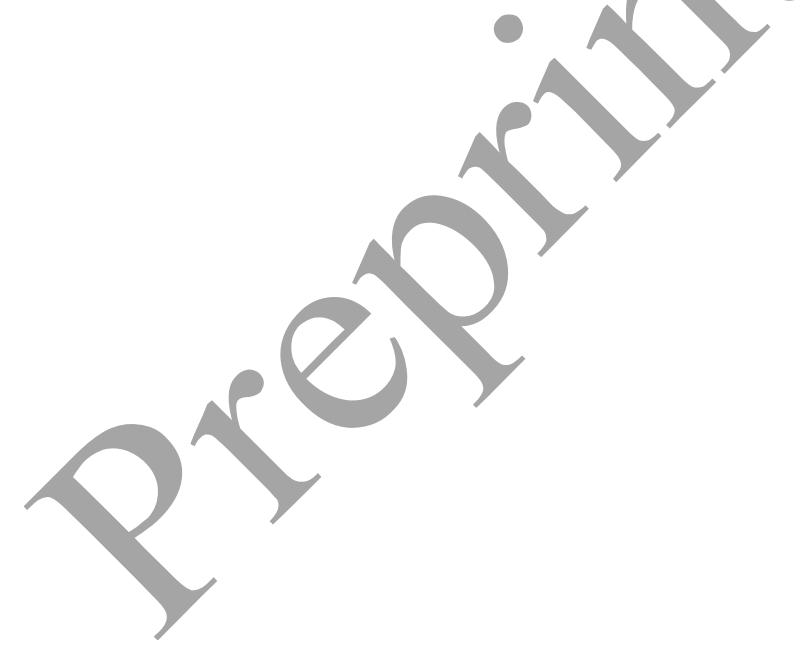


Raw tomatoes

Mashed tomatoes

Tomato concentrate

\begin{tabular}{ccccccccc} 
& \multicolumn{3}{c}{ AOH } & & & \multicolumn{2}{c}{ AME } \\
\cline { 2 - 9 } n & $\begin{array}{c}\text { Positive } \\
\text { Samples } \\
(\mathbf{\%})\end{array}$ & Range* & Mean* & Median* & $\begin{array}{c}\text { Positive } \\
\text { Samples } \\
(\%)\end{array}$ & Range* & Mean* & Median* \\
\hline 34 & $7(21 \%)$ & $336.5-1436.9$ & 1000 & 1165.2 & 0 & ND & ND & ND \\
29 & $4(17 \%)$ & $92.7-107.9$ & 101 & 101.2 & 0 & ND & ND & ND \\
30 & $14(47 \%)$ & $22.6-137.4$ & 45 & 39.0 & 0 & ND & ND & ND
\end{tabular}

456

457

Table 2 .

*The range, the mean and the median were calculated just for positive samples. Units for range, mean and median were $\mu \mathrm{g} / \mathrm{kg}$ of tomato.

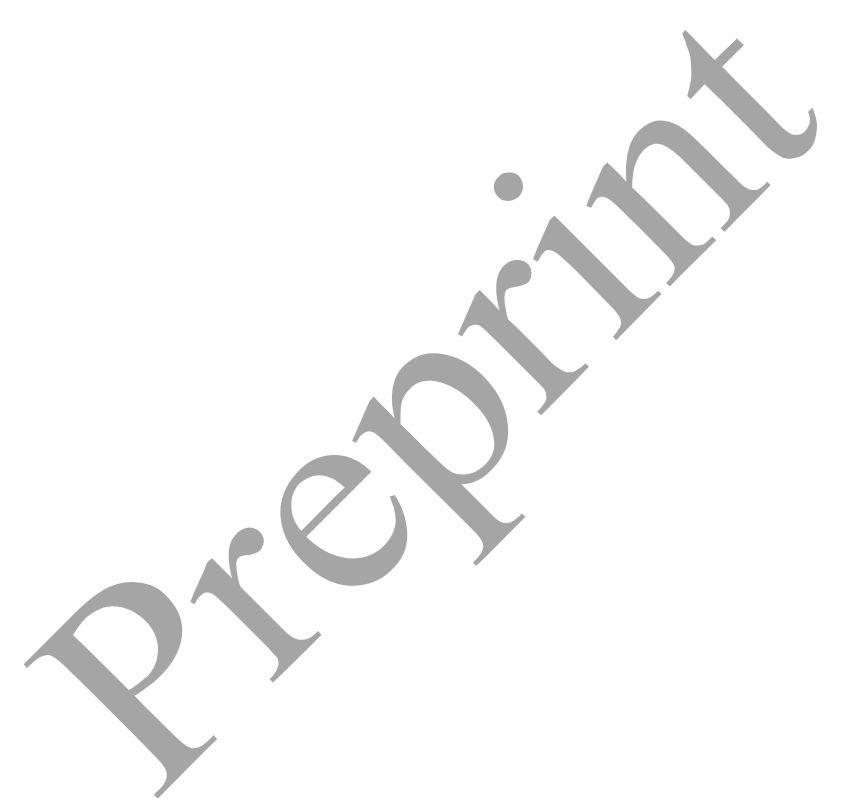

